University of Wollongong

Research Online

Faculty of Engineering and Information

Faculty of Engineering and Information

Sciences - Papers: Part B

Sciences

2017

Tailoring the wettability and mechanical properties of electrospun poly $(\mathrm{L}-$ lactic acid)-poly(glycerol sebacate) core-shell membranes for biomedical applications

Yi Yan

University of Wollongong

Vitor Sencadas

University of Wollongong, victors@uow.edu.au

Tiantian Jin

University of Wollongong, tj122@uowmail.edu.au

Xu-Feng Huang

University of Wollongong, xhuang@uow.edu.au

Jun Chen

University of Wollongong, junc@uow.edu.au

See next page for additional authors

Follow this and additional works at: https://ro.uow.edu.au/eispapers1

Part of the Engineering Commons, and the Science and Technology Studies Commons

Research Online is the open access institutional repository for the University of Wollongong. For further information contact the UOW Library: research-pubs@uow.edu.au 


\title{
Tailoring the wettability and mechanical properties of electrospun poly(L-lactic acid)-poly(glycerol sebacate) core-shell membranes for biomedical applications
}

\begin{abstract}
Tissue and biomedical engineering fields are in constant mutation and in searching for innovative processing techniques capable to tailor the material properties. In this work, poly(l-lactic acid) (PLLA) and elastomeric poly(glycerol sebacate) (PGS) were dissolved in the same solvents and electrospun together, in a single needle system. A core-shell structure where the hydrophilic PGS was placed onto the surface of the hydrophobic PLLA fibre was obtained for elastomeric concentrations up to $25 \mathrm{wt} \%$. It was found that the PLLA:PGS blends are immiscible and the blends present the melting temperatures of the individual polymers. Moreover, their surface properties were deeply influenced by the presence of the PGS, and a superhydrophilic membrane was obtained, after PGS curing at $120^{\circ} \mathrm{C}$ for $48 \mathrm{~h}$. When the concentration of PGS is up to $25 \mathrm{wt} \%$, the blend's Young modulus decreases from $\sim 35.9 \pm 7.1$ to $7.5 \pm 1.4$ MPa and a twofold improvement in the sample stretchability was observed, compared with the pristine PLLA electrospun samples. Finally, in vitro hypothalamus A59 nerve cell culture shows that the core-shell electrospun samples enhanced cell adhesion and proliferation, suggesting that these developed materials have great potentials for nerve regeneration and biomedical engineering applications.

\section{Disciplines}

Engineering | Science and Technology Studies

\section{Publication Details}

Yan, Y., Sencadas, V., Jin, T., Huang, X., Chen, J., Wei, D. \& Jiang, Z. (2017). Tailoring the wettability and mechanical properties of electrospun poly(L-lactic acid)-poly(glycerol sebacate) core-shell membranes for biomedical applications. Journal of Colloid and Interface Science, 508 87-94.
\end{abstract}

\section{Authors}

Yi Yan, Vitor Sencadas, Tiantian Jin, Xu-Feng Huang, Jun Chen, Dongbin Wei, and Zhengyi Jiang 
Tailoring the wettability and mechanical properties of electrospun poly $(\mathrm{L}$ lactic acid)-poly(glycerol sebacate) core-shell membranes for biomedical applications

Yi Yan ${ }^{\mathrm{a}}$, Vitor Sencadas ${ }^{\mathrm{a}, \mathrm{b}, *}$, Tiantian $\operatorname{Jin}^{\mathrm{c}}$, Xufeng Huang ${ }^{\mathrm{c}}$, Jun Chen ${ }^{\mathrm{d}}$, Dongbin Wei ${ }^{\mathrm{a}, \mathrm{e}}$, Zhengyi Jiang ${ }^{\mathrm{a}, *}$

*Corresponding authors e-mail:

Vitor Sencadas, victors@uow.edu.au, Zhengyi Jiang, jiang@uow.edu.au,

${ }^{\mathrm{a}}$ School of Mechanical, Materials, Mechatronic and Biomedical Engineering, University of Wollongong, Wollongong, NSW 2522, Australia

${ }^{\mathrm{b}}$ ARC Center of Excellence for Electromaterials Science, University of Wollongong, 2522 NSW, Australia

'Illawarra Health and Medical Research Institute, University of Wollongong, Wollongong, NSW 2522, Australia

${ }^{\mathrm{d} I n t e l l i g e n t ~ P o l y m e r ~ R e s e a r c h ~ I n s t i t u t e, ~ U n i v e r s i t y ~ o f ~ W o l l o n g o n g, ~ I n n o v a t i o n ~ C a m p u s, ~}$ Squires Way, Fairy Meadow, NSW 2519, Australia

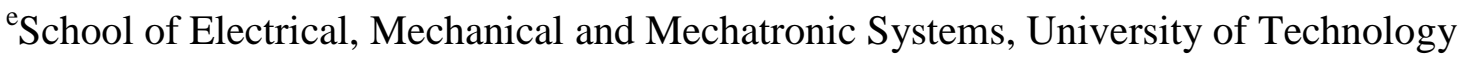
Sydney, Ultimo NSW 2007, Australia

Abstract: Tissue and biomedical engineering fields are in constant mutation and in searching for innovative processing techniques capable to tailor the material properties. In this work, 
poly(L-lactic acid) (PLLA) and elastomeric poly(glycerol sebacate) (PGS) were dissolved in the same solvents and electrospun together, in a single needle system. A core-shell structure where the hydrophilic PGS was placed onto the surface of the hydrophobic PLLA fibre was obtained for elastomeric concentrations up to $25 \mathrm{wt} \%$. It was found that the PLLA:PGS blends are immiscible and the blends present the melting temperatures of the individual polymers. Moreover, their surface properties were deeply influenced by the presence of the PGS, and a superhydrophilic membrane was obtained, after PGS curing at $120{ }^{\circ} \mathrm{C}$ for $48 \mathrm{~h}$. When the concentration of PGS is up to $25 \mathrm{wt} \%$, the blend's Young modulus decreases from $\sim 35.9 \pm 7.1$ to $7.5 \pm 1.4 \mathrm{MPa}$ and a two-fold improvement in the sample stretchability was observed, compared with the pristine PLLA electrospun samples. Finally, in vitro hypothalamus A59 nerve cell culture shows that the core-shell samples produced enhanced cell adhesion and proliferation, suggesting that these developed materials have great potentials for nerve regeneration and biomedical engineering.

Keywords: Electrospun membranes, core-shell electrospun materials, superhydrophilic, nerve tissue engineering

\section{Introduction}

Polymer membranes and scaffolds are widely used in tissue engineering, drug delivery, wound dressing and other biomedical applications [1]. Different techniques are used to produce fibrous membranes, e.g. drawing, template synthesis, phase separation and selfassembly [2, 3]. Nevertheless, electrospinning seems to be the ideal processing method since it is cost-effective and allows the production of one-by-one continuous micro and nanofibers from various polymers, and it can be scaled to industrial production [2, 3]. 
Poly(glycerol sebacate) (PGS), is a ductile and biodegradable elastomer usually obtained by poly-condensation of glycerol and sebacic acid $[4,5]$. The mechanical properties and biodegradation kinetics are deeply influenced by the temperature and time of the synthesis and curing process used. It was reported that the PGS Young modulus increases with the temperature and/or time of curing [6]. Previous studies reported that the curing of the PGS pre-polymer was performed at temperatures in the range of $90-150{ }^{\circ} \mathrm{C}$, and at low pressure, for 24 up to $96 \mathrm{~h}$ [6].

Electrospinning PGS pre-polymer does not lead to the production of polymeric fibres due to their low molecular weight [6, 7], and has a glass transition temperature below room temperature, which promotes flow to the polymer chains, ultimately giving origin to a polymer film [8]. Moreover, cross-linked PGS is insoluble in organic solvents [6, 8], and is not possible to produce a suitable solution for electrospinning.

Core-shell electrospinning can use an electrospinnable polymer as a carrier to produce electrospun fibres of non-electrospinnable materials. Due to the high temperatures used for the PGS curing process, the carrier material needs to be thermally stable and with a melting transition above the temperature used for the PGS curing.

Different strategies are being developed to produce PGS electrospun membranes, based in the blend of the PGS pre-polymer blend with a suitable carrier, that can form fibres and facilitates the fibre formation. Jeffries et al. [8] blended PGS pre-polymer with poly(vinyl alcohol) (PVA) in a common 1,1,1,3,3,3-hexafluoroisopropanol (HFIP) solvent, and the electrospun sample was submitted to a cross-linking procedure, followed by removal of the carrier polymer. PVA:PGS overall fibre diameter was around $2.8 \mu \mathrm{m}$, with high extensions of fibre fusion, especially between their contact points.

Poly(methyl methacrylate) (PMMA) [9] and gelatin [10] were also used as carrier materials to form electrospun PGS fibres membranes. When PMMA was used, the average fibre 
diameter of the electrospun membranes was $\sim 200 \mathrm{~nm}$, with a strong hydrophobic behaviour, which was probably due to the presence of higher amounts of PMMA on the surface of the fibres [9]. When gelatin was used as a carrier polymer for the PGS pre-polymer, an increase of the average diameter of electrospun fibre and hydrophilicity was observed [10]. Poly (L-lactic acid) (PLLA) was also used as a carrier polymer to produce the PGS electrospun fibre membranes. Xu et al. [11] demonstrated that core-shell electrospinning was a feasible technique to immobilise the PGS pre-polymer on the surface of the PLLA fibres. In their work, they electrospun the PLLA in the outer layer of the needle and the PGS prepolymer was fed onto the shell of the needle, immobilising the PGS prepolymer in the core of the produced PLLA fibres. After cross-linking at $130{ }^{\circ} \mathrm{C}$ for three days, fibres with an average diameter of $10 \mu \mathrm{m}$ were obtained, and the membranes produced presented high extensions of fibre fusion.

The objective of this work is to provide a novel, reliable and reproducible method to produce electrospun PLLA:PGS membranes, where the PGS elastomer is immobilised on the surface of the PLLA fibre. The PGS prepolymer was blended with the PLLA solution and electrospun together in a single needle system, followed by PGS cross-linking. The effect on fibre average diameter of the PGS pre-polymer concentration in the electrospinning solution was systematically assessed. Moreover, the influences of the PGS layer on the fibre membranes wettability, thermal and mechanical properties were studied. Finally, biological assays demonstrated that the core-shell samples produced have great potentials for nerve regeneration and biomedical engineering.

\section{Experimental}

2.1. Materials: Medical degree poly(L-lactic acid) (PLLA, Purasorb PL18, $\mathrm{M}_{\mathrm{w}}=217-225$

kDa, from Corbion Netherlands), glycerol, sebacic acid, N,N-dimethylformamide (DMF), 
dichloromethane (DMC), hexane and formamide (from Sigma-Aldrich Australia) were used as received.

2.2. PGS synthesis: For PGS prepolymer preparation, equimolar glycerol and sebacic acid were mixed in the flask at $120{ }^{\circ} \mathrm{C}$ under argon for $24 \mathrm{~h}$, and used without further purification during the materials processing. PGS prepolymer was cured in the oven (Shel Lab, Model 1401D) at $120^{\circ} \mathrm{C}$ under vacuum, for $48 \mathrm{~h}$ to prepare cross-linked PGS films.

2.3. PLLA and PGS:PLLA electrospun membranes: PLLA pellets were dissolved in a mixed solvent of DMF and DMC (3/7 v/v) to achieve a solution with a final polymer concentration of $10 \mathrm{wt} \%$. PGS:PLLA solutions were prepared by adding the desired amounts of PGS prepolymer (0, 25, 40 and $50 \mathrm{wt} \%)$ to the PLLA solution prepared according to the method described above. The PLLA and PGS:PLLA were dissolved at room temperature with the help of a magnetic stirrer (Velp, Model MST) until complete dissolution. After, the solution was transferred to a commercial $10 \mathrm{~mL}$ glass syringe fitted with a steel needle (inner diameter $500 \mu \mathrm{m})$. Electrospinning system was set at $1.67 \mathrm{kV} / \mathrm{cm}$ with a high voltage power supply (Gamma High Voltage). A syringe pump (KDScientific) fed the polymer solutions into the needle tip at the rate of $0.5 \mathrm{~mL} / \mathrm{h}$. Randomly aligned PGS:PLLA fibres were collected in ground collecting plate $15 \mathrm{~cm}$ away from the needle tip. All experiments were conducted at $21 \pm 2{ }^{\circ} \mathrm{C}$ and a relative humidity of $43 \pm 5 \%$.

PGS cross-linking was performed as per the method described above for the PGS film. Briefly, the samples were placed inside a vacuum oven at $120^{\circ} \mathrm{C}$, for $48 \mathrm{~h}$. The same procedure was also performed to a neat PLLA to understand the effect of the curing temperature. 
2.4. Materials characterisations: Surface morphology of the samples was analysed by scanning electron microscopy (JEOL-6490LV), after deposition of a thin layer of platinum $(10 \mathrm{~nm})$ on the sample surface (Dynavac Sputter Coater). For sample cross-section analysis, the material was immersed in the liquid nitrogen for around 10 min for thorough cooling, followed by breaking material, and then attached to the cross-section holder for platinum coating, followed by morphology observation using JEOL JSM-7500.

Fibres diameters were measured using Image [12] based on the surface morphology SEM images with at least 100 fibres included for each sample.

Thermal gravimetric analysis (TGA) was carried out using TA instruments TGA (Q500). Samples of around $10 \mathrm{mg}$ were prepared and heated from room temperature to $600{ }^{\circ} \mathrm{C}$ at $20{ }^{\circ} \mathrm{C} / \mathrm{min}$. Differential scanning calorimetry (DSC) experiments were conducted by TA instruments (Q100). Samples of around $5 \mathrm{mg}$ were placed inside $50 \mu \mathrm{L}$ aluminium pans, and heated between -50 and $200{ }^{\circ} \mathrm{C}$ at a heating rate of $10^{\circ} \mathrm{C} / \mathrm{min}$. All experiments (TGA and DSC) were performed under a nitrogen atmosphere.

The PGS:PLLA electrospun samples and PGS film, were cut into dog-bone shape of $2 \mathrm{~mm}$ wide and $18 \mathrm{~mm}$ for the gauge length. Quasi-static tensile tests were conducted by Shimadzu EZ-L mechanical tester using the $2 \mathrm{~N}$ load cell (for PGS) and $10 \mathrm{~N}$ load cell (for fibres) with the cross-head speed of $1 \mathrm{~mm} / \mathrm{min}$ at room temperature. At least three measurements were conducted for each sample.

2.5. Samples wettability: Water contact angle was measured using goniometer (DataPhysics OCA 20 contact angle system from Germany) to study the surface properties of the fibrous samples. At least five measurements were done for each sample. 
2.6. In vitro biocompatibility: All the fibrous samples were cut into round shape with a diameter of $6.4 \mathrm{~mm}$. To remove the possibly left unreacted monomers, the samples were immersed in ethanol for around $2 \mathrm{~h}$, followed by washing twice with phosphate-buffered saline (PBS, $\mathrm{pH}=7.4$ ) and then mechanically stirred in PBS for $12 \mathrm{~h}$. Samples were sterilised with ultra violet light $(\lambda=254 \mathrm{~nm})$ for 20 min each side before cell seeding. Adult mouse hypothalamus neurons A59 cell line (mHypoA-59, Cellutions biosystems) was used to examine the in vitro biocompatibility. The samples were put on the bottom of the wells of 96 well plate and 1000 cells were seeded for each well. Cells were incubated in $100 \mu \mathrm{L}$ Dulbecco’s modified Eagle medium (DMEM) (D5796, Sigma-Aldrich) at $37^{\circ} \mathrm{C}$ and $5 \% \mathrm{CO}_{2}$ for 5 days with the medium refreshed on day 2 and day 4 . The cell culture wells with cells only were used as control.

Methotrexate (MTX) assay was performed on day 1, day 3 and day 5. $20 \mu \mathrm{L}$ MTX solution was added into each well and the culture plate was incubated at the same condition for $2 \mathrm{~h}$. Supernatants were collected to detect absorbance value via using Spectromax plus 384 plate reader. Cell viability of the samples after 1,3 and 5 days was obtained by comparing the optical density values of the sample groups to control group.

The outgrowth and morphology of the cells were observed by JEOL-6490LV. On day 1, day 3 and day 5, cells were fixed with 4\% paraformaldehyde in Dulbecco's PBS for 20 min at room temperature and then the samples were dehydrated by immersion in a series of ethanol solutions (50, 75, 90, 95 and 100\%), dried at room temperature and coated with a platinum thin film of $10 \mathrm{~nm}$ on the surface for SEM observation.

2.7. Statistics and Data Analysis: Statistical analysis was performed by a Kruskal-Wallis test with a Dunn`s post hoc test, using GraphPad Prism 6.01 (GraphPad Software, Inc.). Statistical differences were assigned to the experimental groups with $\mathrm{p}<0.05$. 


\section{Results and discussion}

\subsection{Morphology and structural analysis}

In this work, PLLA polymer and PGS prepolymer were mixed in the same solution and electrospun together. PLLA electrospun fibre mats showed a random distribution and cylindrical, smooth fibres, in the absence of bead defects, and the thermal annealing at $120{ }^{\circ} \mathrm{C}$, and during $48 \mathrm{~h}$ (simulating the PGS curing procedure) does not influence the fibre morphology (Figure 1a). Moreover, when $25 \mathrm{wt} \%$ PGS prepolymer is added to the polymer solution and electrospun together, smooth fibres without the presence of bead defects are obtained (Figure 1b). However, when the prepolymer concentration increases above 25 wt\%, spots of prepolymer randomly distributed within the fibre membrane where observed, increasing their size with the increase of the amount of prepolymer added to the electrospun solution (Figure 1c and d).

The PGS curing procedure at $120^{\circ} \mathrm{C}$ didn't alter the fibre morphology, or removed the PGS prepolymer spots from the membrane. On the other hand, for the sample with $25 \mathrm{wt} \%$ PGS, it was observed that the PGS polymer was dragged by the PLLA polymer during electrospinning, and after curing, the elastomeric material was placed on outside, and the PLLA was on the core of the electrospun fibre, creating a core-shell structure, in a single electrospinning step (Figure 1e). Usually, core-shell fibre area obtained by feeding two independent solutions through a core-shell needle, where one polymer is fed in the core of the needle and the other is fed on the outside chamber of the needle, and both solutions, only met on the edge of the needle $[13,14]$.

The processing method developed to electrospun the PGS:PLLA polymer, creates a coreshell structure in a continuous manner without clogging the needle tip and avoids the search for solvent compatible for electrospinning two different solutions [14]. 
The influence of the amount of PGS prepolymer in the final electrospun fibre diameter was measured over 100 fibres from different SEM images with the help of Image J. It was observed that the pristine PLLA electrospun fibres had an average diameter of $746 \pm 274 \mathrm{~nm}$, and with the incorporation of PGS prepolymer, a reduction in the fibre average diameter down to $332 \pm 103 \mathrm{~nm}$ was observed for the sample with $50 \mathrm{wt} \%$ PGS (Figure 1f). This reduction in the fibre diameter shows that the PGS prepolymer probably affects charge density of the solutions. PGS prepolymer can be ionised and carry more charges, stretching the polymer droplet even further, resulting in a decrease of the average fibre diameter of the core-shell structure. Similar effect was observed for mixture of polymers with ceramic fillers, e.g. PLLA with glass reinforced hydroxyapatite [15], or for PLLA zeolite nanocomposite samples [16]. In comparison with other works, the average diameter of the electrospun coreshell fibres obtained in this work, was remarkably thinner. Previous works reported a fibre average diameter between $\sim 2$ and $8 \mu \mathrm{m}$ for PGS electrospun membranes produced with PCL [17] or PVA [8] as carrier polymers.

\section{Figure 1:}

The effect of the PLLA:PGS electrospinning processing parameters can be monitored by Fourier transform infrared (FTIR) spectroscopy in Attenuated Total Reflectance (ATR). PLLA absorption band at $1757 \mathrm{~cm}^{-1}$ can be assigned to the $\mathrm{C}=\mathrm{O}$, attributed to the amorphous regions of the polymer chains, while the characteristic absorption band for the amorphous $\alpha$ and $\alpha^{\prime}$ was observed at $1183 \mathrm{~cm}^{-1}\left(v_{a s}(C-O-C)+r_{s}\left(C_{3}\right)\right)$ and $1092 \mathrm{~cm}^{-1}$ $\left(v_{S}(C-O-C)\right)$, respectively [18]. PGS elastomer presents a characteristic absorption band at $1736 \mathrm{~cm}^{-1}$, associated to the stretching of the carbonyl ester group $(\mathrm{C}=\mathrm{O})$ and the absorption band at $1185 \mathrm{~cm}^{-1}$ is due to the $\mathrm{C}$ - $\mathrm{O}$ in-plane bending vibration [19]. Moreover, 
there is also a broad absorption band between 3300 and $3800 \mathrm{~cm}^{-1}$ for hydroxyl bond stretch vibration [20].

The electrospun fibre membranes present the characteristic absorption bands of the core PLLA and the shell PGS. No new absorption band was totally suppressed or new ones appeared in the core-shell PLLA:PGS electrospun mats, suggesting that the blends between these polymers are difficult to occur. Moreover, for the PLLA:PGS, two shoulders were observed between the $1757 \mathrm{~cm}^{-1}(\mathrm{C}=\mathrm{O})$ of PLLA and the carbonyl ester absorption band of PGS $\left(1736 \mathrm{~cm}^{-1}\right)$, which is in line with the spectra of the pure polymers (Figure 2).

\section{Figure 2:}

\subsection{Thermal properties}

The evolution of the thermal degradation of the PLLA, PGS and PLLA:PGS was assessed by thermogravimetric analysis (TGA). PLLA presents a major weight loss in the temperature range of $250-350{ }^{\circ} \mathrm{C}$, and no water adsorption was observed around $100{ }^{\circ} \mathrm{C}$ (Figure 3a). Moreover, PGS polymer presented a single degradation process between 350 - $500{ }^{\circ} \mathrm{C}$, and no significant dehydration process was observed at lower temperatures $\left(\leq 100{ }^{\circ} \mathrm{C}\right.$, Figure 3a) [21]. PLLA:PGS core-shell fibres presented two thermal degradation steps; the first one between 200 and $350{ }^{\circ} \mathrm{C}$, associated to the degradation process of the PLLA polymer core [6, 17], and the second one between $350-500{ }^{\circ} \mathrm{C}$, due to the thermal degradation of the PGS shell layer. It was also noticed that for the core-shell PLLA:PGS samples, the $T_{\text {onset }}$, calculated by extending the pre-degradation portion of the curve to the point of the interception with a line drawn as a tangent to the steepest portion of the mass curve occurring during degradation, started a lower temperatures for the PLLA:PGS fibres, when compared to 
the pristine materials (Table 1), suggesting that the thermal stability of the core-shell membranes is lower when compared to the neat polymer constituents, which is probably due to interaction between the PLLA core and PGS filler at the interface of the core-shell fibres (Figure 3a).

\section{Figure 3:}

Differential scanning calorimetry was performed to the different samples to assess the evolution of the glass transition $\left(T_{g}\right)$, cold crystallisation and melting behaviour of the pristine materials and core-shell fibre samples (Figure 3b). PLLA pristine fibres present a glass transition around $55^{\circ} \mathrm{C}$, followed by the melting of the crystalline lamellas around $150{ }^{\circ} \mathrm{C}$. The absence of the cold-crystallisation region in the electrospun PLLA area is due to the annealing procedure performed to the samples at $120^{\circ} \mathrm{C}$ for $48 \mathrm{~h}$ (Figure 3b) [18, 22]. Furthermore, pristine PGS samples show a melting temperature $\left(T_{m}\right)$ around $5 \mathrm{C}^{\circ}$ (Figure $3 \mathrm{~b}$ ) with an associated enthalpy of fusion of $27.1 \mathrm{~J}^{-g^{-1}}$, which is in the range of the values found in literature [23]. The PLLA:PGS fibre membranes present the characteristic $T_{m}$ of the PGS and PLLA polymers, and no significant shift in the $T_{g}$ of the PLLA core material is noticed in Figure 3b. Our results suggest that the PLLA and PGS polymers do not interact between each other, which corroborate the SEM results (Figure 1), where a clear PGS hollow fibre was observed.

The degree of crystallinity $(\Delta X)$ of the pristine PLLA and PLLA:PGS electrospun samples, after cross-linking under vacuum for $48 \mathrm{~h}$ and at $120^{\circ} \mathrm{C}$ was calculated with the following equation: 


$$
\Delta X=\frac{\Delta H_{m}}{\Delta H_{m}^{0}}
$$

where, $\Delta H_{m}$ is the sample melting enthalpy, and $\Delta H_{m}^{0}$ the enthalpy for a $100 \%$ crystalline PLLA sample (93 J.g $\left.{ }^{-1}\right)$ [21, 22]. After cross-linking, the PLLA polymer in the PLLA:PGS electrospun samples presented a crystallinity degree of $44 \%$, independently of the amount of PGS in the shell of the core-shell fibre structure. The value calculated through Equation 1 is similar to the value reported for the PLLA electrospun samples annealed for $24 \mathrm{~h}$ at $140{ }^{\circ} \mathrm{C}$ [24].

Table 1: Onset temperatures and weight residue of PLLA and PLLA:PGS samples.

\begin{tabular}{lll}
\hline $\begin{array}{l}\text { Amount of PGS } \\
(\mathbf{w t} \%)\end{array}$ & \multicolumn{1}{c}{$\boldsymbol{T}_{\text {onset }}$} & $\begin{array}{l}\text { Residues at } 400{ }^{\circ} \mathbf{C} \\
\left({ }^{\circ} \mathbf{C}\right)\end{array}$ \\
\hline 0 & 305.2 & 2 \\
25 & 255.4 & 19 \\
40 & 248.6 & 30 \\
50 & 254.5 & 36 \\
100 & 398.6 & 83 \\
\hline
\end{tabular}

\subsection{Mechanical properties}

The mechanical behaviour of scaffolds is a key parameter to evaluate the suitability of the developed material for biomedical applications. In this work, quasi-static mechanical analysis was performed to the PLLA, PGS and to the PLLA:PGS core-shell electrospun membranes. Table 2 lists the values for the Young's modulus $(E)$, the stress at break $\left(\sigma_{\text {break }}\right)$ and strain at break $\left(\varepsilon_{\text {break }}\right)$ for all cured membranes. Our results suggest that when an amount of PGS up 
to $40 \mathrm{wt} \%$ is added to the electrospinning solution, the ductility of the polymeric blend is improved compared to the pristine PLLA electrospun fibres. For instance, the cured PLLA:PGS membranes with 25 and $40 \mathrm{wt} \%$ show an approximately 2-fold increase of the strain at break when compared to the pristine PLLA. Moreover, the incorporation of the PGS into the PLLA:PGS membranes leads to a decrease of the $\sigma_{\text {break }}$ when $25 \mathrm{wt} \%$ PGS is incorporated into the polymer solution, however, for higher PGS concentrations, there is an increase of the stress at break, probably due to the reduction of the pore size and the presence of the PGS agglomerates, present not only on the surface of the material but also through the bulk of the membrane (Figure 1). Frydrych et al. [25] found that the amount of PGS added to the PLLA:PGS scaffold influences the polymeric blend mechanical behaviour. In their work, they found that the incorporation of higher amounts of PGS, led to a reduction of the pore diameter, and consequently to an increase of the mechanical stiffness and reduction of the strain at break of the prepared scaffolds.

Table 2: Mechanical properties of electrospun fibres and PGS.

\begin{tabular}{lccc}
\hline $\begin{array}{l}\text { Amount of } \\
\text { PGS }\end{array}$ & $\boldsymbol{\sigma}_{\text {break }}(\mathbf{M P a})$ & $\boldsymbol{\varepsilon}_{\text {break }}$ & $\boldsymbol{E}$ \\
$\mathbf{( w t \% )}$ & $(\mathbf{\% )}$ & $\mathbf{( M P a )}$ \\
\hline 0 & $2.6 \pm 0.3$ & & $35.9 \pm 7.1$ \\
25 & $1.1 \pm 0.2$ & $29 \pm 6$ & $7.2 \pm 1.4$ \\
40 & $6.8 \pm 0.2$ & $66 \pm 8$ & $379.0 \pm 29.9$ \\
50 & $6.7 \pm 1.1$ & $52 \pm 9$ & $336.2 \pm 25.8$ \\
100 & $0.18 \pm 0.01$ & $13 \pm 5$ & $0.08 \pm 0.01$ \\
\hline
\end{tabular}




\subsection{Wettability properties}

Membrane surface properties and wettability are important features to assess the potential of the developed membranes for biomedical applications, because they play a vital role in cell proliferation and adhesion [26]. The average water contact angle (WCA) measured for the PLLA electrospun fibres was $125 \pm 2^{\circ}$, revealing a strong hydrophobic behaviour, probably due to the overall membrane roughness [15, 22]. Moreover, the PGS polymer film showed to have a hydrophilic behaviour, with a WCA of $42 \pm 3^{\circ}$. Interestingly, for all PLLA:PGS electrospun samples, the water drop placed on top of the membrane was not stable and the membrane was capable of uptake of almost instantaneously the liquid. The results reveal that the incorporation of PGS onto the shell of the PLLA:PGS fibres improves the wettability of the electrospun membranes significantly, and no WCA measurements and drop penetration test are possible to perform on this superhydrophilic membranes. Similar results were reported for poly(caprolactone)-PGS electrospun fibres, and the superhydrophilic behaviour was attributed to the presence of the hydroxyl groups attached to the backbone of the PGS polymer, and due to the penetration and capillary effect of the porous membrane [20].

\subsection{In vitro biocompatibility of PLLA:PGS fibrous blends}

Biomaterial-cell interaction is affected by material roughness, surface free energy, hydrophilicity, among other material properties [27]. Thus, for tissue engineering and biomedical applications, it is highly desirable to have hydrophilic surfaces [22, 27]. In the present work, it was observed that the immobilisation of the PGS polymer onto the surface of the PLLA fibre gives origin to superhydrophilic membranes, capable to almost instantaneously uptake water.

A59 neuronal cell viability in PLLA and PLLA:PGS fibrous membranes was evaluated in vitro by using MTX assay, which gives an indication of cell metabolic activity. Cells were 
seeded on the surface of the fibrous samples, and grown for for one, three and five days, after which proliferation was assessed by measuring the metabolic activity of the cells with an MTX assay (Figure 4). After 1one and three days of incubation, the A59 neuronal cells cultured on the PLLA and PLLA:PGS fibrous mats showed a proliferation profile to the one obtained with the control, where the cells were cultured in a standard polystyrene (TCPS) wells. After five days of incubation, proliferation of cells grown on PLLA and PLLA:PGS electrospun membranes was slightly lower when compared to the control (Figure 4). Nevertheless, no significant differences were detected between the different PLLA and PLLA:PGS samples, which suggests that the presence of the hydrophilic PGS shell is suitable material for biomedical applications.

\section{Figure 4:}

The micrographs recorded with SEM, showed spread cells on the membrane surface (Figure 5), displaying the characteristic flat phenotype, with extended cytoplasm, and appearing to interact and associate with surrounding fibres (Figure 5) [28, 29]. Even at day 1, the morphology of the cells was dominated by cells in advanced stage of adhesion (Figure 5). It was possible to observe that cells were spread on the surface of the fibres, adopting a polygonal morphology, and adhered to the surface with lamellipodia and filopodia extensions. It was observed that in the case of the PLLA:PGS fibres, the cells spread out and elongated to form long processes of neurite varicosities, tried to interact with the electrospun fibres and communicate with neighbour cells through the nanofibers (Figure 5), suggesting that the developed membranes have the ability to induce the differentiation of A59 cells into neuronlike cells, even in the absence of growth factor or chemical treatments. 
In the first few hours after culture, cells often attempt to explore the surroundings by dynamically extruding and retracting thin filopodia protrusions in arbitrary directions. As the cell begins to flatten, on the surface of the fibrous membranes, wider lamellipodia protrusions at the leading edge of the cell starts to appear and actively propel the cell front forward [30]. After 5 days, cells actively proliferated, and the establishment of intercellular contacts were identified within PLLA and PLLA:PGS electrospun membranes (Figure 5).

\section{Figure 5:}

\section{Conclusions}

PLLA and PLLA:PGS fibre structure was obtained by a single needle electrospinning system, which is versatile, reproducible and can be readily upscale, without compromising the membrane characteristics. It was found that the incorporation of PGS amounts up to $25 \mathrm{wt} \%$, individual and defect free fibres were obtained, where the PGS shell wraps the PLLA core, while for higher amounts of PGS pre-polymer present in the electrospun solution, randomly dispersed agglomerates of PGS film was observed in the membrane samples. Further, the blends between the PGS pre-polymer and the PLLA are immiscible and a melting transition can be observed at $\sim 5^{\circ} \mathrm{C}$ for the PGS shell, and at $\sim 150{ }^{\circ} \mathrm{C}$ corresponding to the PLLA fibre core melting. When PGS is added with a concentration up to $25 \mathrm{wt} \%$, the blend's Young modulus decrease from $\sim 35.9 \pm 7.1 \mathrm{MPa}$ (pristine PLLA) down to $7.5 \pm 1.4 \mathrm{MPa}$ (core-shell membrane), and a two-fold improvement in the sample stretchability was observed, when compared with the pristine PLLA electrospun samples. Moreover, by incorporating the PGS polymer on the surface of the core-shell PLLA:PGS fibres, the wettability of the materials is enhanced, and a superhydrophilic material was achieved for the sample with 
small amount of PGS. Finally, in vitro hypothalamus A59 nerve cell culture shows that the core-shell samples produced enhanced cell adhesion and proliferation, suggesting that these materials have great potentials for nerve regeneration and biomedical engineering.

\section{Acknowledgements}

The authors acknowledge financial support from China Scholarship Council (CSC), UPA from University of Wollongong and Australian Research Council (ARC) for Future Fellowship (Z.Y. Jiang). The authors acknowledge use of facilities within the UOW Electron Microscopy Centre and the Australian National Nanofabrication Facility - Materials node for equipment use. The authors acknowledge Mr. Jiangshan Zhang and Dr. Tony Romeo for SEM imaging, Ms. Hongqin Wang, Dr. Xiaoqi Chen, Dr. Qiang Fu and Ms. Zehra Boz for their help of cell culture. V. Sencadas thanks support from the COST Actions MP1206: “Electrospun nano-fibres for bio inspired composite materials and innovative industrial applications” and MP1301: “New Generation Biomimetic and Customized Implants for Bone Engineering”.

\section{References}

[1] U. Boudriot, R. Dersch, A. Greiner, J.H. Wendorff, Electrospinning Approaches Toward Scaffold Engineering-A Brief Overview, Artificial Organs 30(10) (2006) 785-792.

[2] Z.-M. Huang, Y.Z. Zhang, M. Kotaki, S. Ramakrishna, A review on polymer nanofibers by electrospinning and their applications in nanocomposites, Composites Science and Technology 63(15) (2003) 2223-2253.

[3] J.L. Ifkovits, R.F. Padera, J.A. Burdick, Biodegradable and radically polymerized elastomers with enhanced processing capabilities, Biomed Mater 3(3) (2008). 
[4] Y. Yan, V. Sencadas, J. Zhang, G. Zu, D. Wei, Z. Jiang, Processing, characterisation and electromechanical behaviour of elastomeric multiwall carbon nanotubes-poly (glycerol sebacate) nanocomposites for piezoresistive sensors applications, Composites Science and Technology 142 (2017) 163-170.

[5] Y. Wang, G.A. Ameer, B.J. Sheppard, R. Langer, A tough biodegradable elastomer, Nature biotechnology 20(6) (2002) 602-6.

[6] R. Rai, M. Tallawi, A. Grigore, A.R. Boccaccini, Synthesis, properties and biomedical applications of poly(glycerol sebacate) (PGS): A review, Progress in Polymer Science 37(8) (2012) 1051-1078.

[7] K.G. Akhilesh, N. Mehdi, S. Shilpa, K. Ali, Anisotropic poly (glycerol sebacate)-poly ( $\epsilon$ -caprolactone) electrospun fibers promote endothelial cell guidance, Biofabrication 7(1) (2015) 015001.

[8] E.M. Jeffries, R.A. Allen, J. Gao, M. Pesce, Y. Wang, Highly elastic and suturable electrospun poly(glycerol sebacate) fibrous scaffolds, Acta Biomaterialia 18 (2015) 30-39. [9] J. Hu, D. Kai, H. Ye, L. Tian, X. Ding, S. Ramakrishna, X.J. Loh, Electrospinning of poly(glycerol sebacate)-based nanofibers for nerve tissue engineering, Materials Science and Engineering: C 70, Part 2 (2017) 1089-1094.

[10] J.L. Ifkovits, J.J. Devlin, G. Eng, T.P. Martens, G. Vunjak-Novakovic, J.A. Burdick, Biodegradable Fibrous Scaffolds with Tunable Properties Formed from Photo-CrossLinkable Poly(glycerol sebacate), ACS Applied Materials \& Interfaces 1(9) (2009) 18781886.

[11] B. Xu, Y. Li, X. Fang, G.A. Thouas, W.D. Cook, D.F. Newgreen, Q. Chen, Mechanically tissue-like elastomeric polymers and their potential as a vehicle to deliver functional cardiomyocytes, Journal of the Mechanical Behavior of Biomedical Materials 28 (2013) 354-365. 
[12] C.A. Schneider, W.S. Rasband, K.W. Eliceiri, NIH Image to ImageJ: 25 years of image analysis, Nat Meth 9(7) (2012) 671-675.

[13] B. Xu, B. Rollo, L.A. Stamp, D. Zhang, X. Fang, D.F. Newgreen, Q. Chen, Non-linear elasticity of core/shell spun PGS/PLLA fibres and their effect on cell proliferation, Biomaterials 34(27) (2013) 6306-6317.

[14] S. Ramakrishna, K. Fujijhara, W.-E. Teo, T.-C. Lim, Z. Ma, An Introduction to Electrospinning and Nanofibers, World Scientific2005.

[15] D. Santos, C.O. Correia, D.M. Silva, P.S. Gomes, M.H. Fernandes, J.D. Santos, V. Sencadas, Incorporation of glass-reinforced hydroxyapatite microparticles into poly(lactic acid) electrospun fibre mats for biomedical applications, Materials Science and Engineering: C 75 (2017) 1184-1190.

[16] H. Zhou, K.-W. Kim, E. Giannelis, Y.L. Joo, Nanofibers from Polylactic Acid Nanocomposites: Effect of Nanoclays on Molecular Structures, in: Darrell H. Reneker, H. Fong (Eds.), Polymeric Nanofibers, American Chemical Society2006, pp. 217-230. [17] A.K. Gaharwar, A. Patel, A. Dolatshahi-Pirouz, H. Zhang, K. Rangarajan, G. Iviglia, S.R. Shin, M.A. Hussain, A. Khademhosseini, Elastomeric nanocomposite scaffolds made from poly(glycerol sebacate) chemically crosslinked with carbon nanotubes, Biomaterials Science (2015).

[18] R. Clarisse, S. Vitor, C. Carlos Miguel, R. José Luís Gómez, L.-M. Senentxu, Tailoring the morphology and crystallinity of poly(L-lactide acid) electrospun membranes, Science and Technology of Advanced Materials 12(1) (2011) 015001.

[19] H.M. Aydin, K. Salimi, M. Yilmaz, M. Turk, Z.M.O. Rzayev, E. Pişkin, Synthesis and characterization of poly(glycerol-co-sebacate-co-e-caprolactone) elastomers, Journal of Tissue Engineering and Regenerative Medicine 10(1) (2016) E14-E22. 
[20] S. Salehi, M. Fathi, S.H. Javanmard, T. Bahners, J.S. Gutmann, S. Ergün, K.P. Steuhl, T.A. Fuchsluger, Generation of PGS/PCL Blend Nanofibrous Scaffolds Mimicking Corneal Stroma Structure, Macromolecular Materials and Engineering 299(4) (2014) 455-469. [21] V. Sencadas, C.M. Costa, G. Botelho, C. Caparrós, C. Ribeiro, J.L. Gómez-Ribelles, S. Lanceros-Mendez, Thermal Properties of Electrospun Poly(Lactic Acid) Membranes, Journal of Macromolecular Science, Part B 51(3) (2012) 411-424.

[22] T.A.M. Valente, D.M. Silva, P.S. Gomes, M.H. Fernandes, J.D. Santos, V. Sencadas, Effect of Sterilization Methods on Electrospun Poly(lactic acid) (PLA) Fiber Alignment for Biomedical Applications, ACS Applied Materials \& Interfaces 8(5) (2016) 3241-3249.

[23] I.H. Jaafar, M.M. Ammar, S.S. Jedlicka, R.A. Pearson, J.P. Coulter, Spectroscopic evaluation, thermal, and thermomechanical characterization of poly(glycerol-sebacate) with variations in curing temperatures and durations, Journal of Materials Science 45(9) (2010) 2525-2529.

[24] J.C. Dias, C. Ribeiro, V. Sencadas, G. Botelho, J.L.G. Ribelles, S. Lanceros-Mendez, Influence of fiber diameter and crystallinity on the stability of electrospun poly(l-lactic acid) membranes to hydrolytic degradation, Polymer Testing 31(6) (2012) 770-776.

[25] M. Frydrych, B. Chen, Large three-dimensional poly(glycerol sebacate)-based scaffolds - a freeze-drying preparation approach, Journal of Materials Chemistry B 1(48) (2013) 66506661.

[26] M. Raul, C. André da, S. Vitor, G.-A. Carmen, M.C. Carlos, P. Jorge, G. Andreia, L.-M. Senentxu, R.-C. José Carlos, C. Margarida, Electrospun silk-elastin-like fibre mats for tissue engineering applications, Biomedical Materials 8(6) (2013) 065009.

[27] B. Gupta, N. Anjum, Plasma and Radiation-Induced Graft Modification of Polymers for Biomedical Applications, in: H. Kausch, N. Anjum, Y. Chevolot, B. Gupta, D. Léonard, H.J. 
Mathieu, L.A. Pruitt, L. Ruiz-Taylor, M. Scholz (Eds.), Radiation Effects on Polymers for Biological Use, Springer Berlin Heidelberg, Berlin, Heidelberg, 2003, pp. 35-61.

[28] D. Santos, D.M. Silva, P.S. Gomes, M.H. Fernandes, J.D. Santos, V. Sencadas, Multifunctional PLLA-ceramic fiber membranes for bone regeneration applications, Journal of Colloid and Interface Science (2017).

[29] M.S. Laranjeira, M.H. Fernandes, F.J. Monteiro, Innovative macroporous granules of nanostructured-hydroxyapatite agglomerates: Bioactivity and osteoblast-like cell behaviour, Journal of Biomedical Materials Research Part A 95A(3) (2010) 891-900.

[30] R. Kaunas, A. Zemel, Cell and Matrix Mechanics, CRC Press2014. 

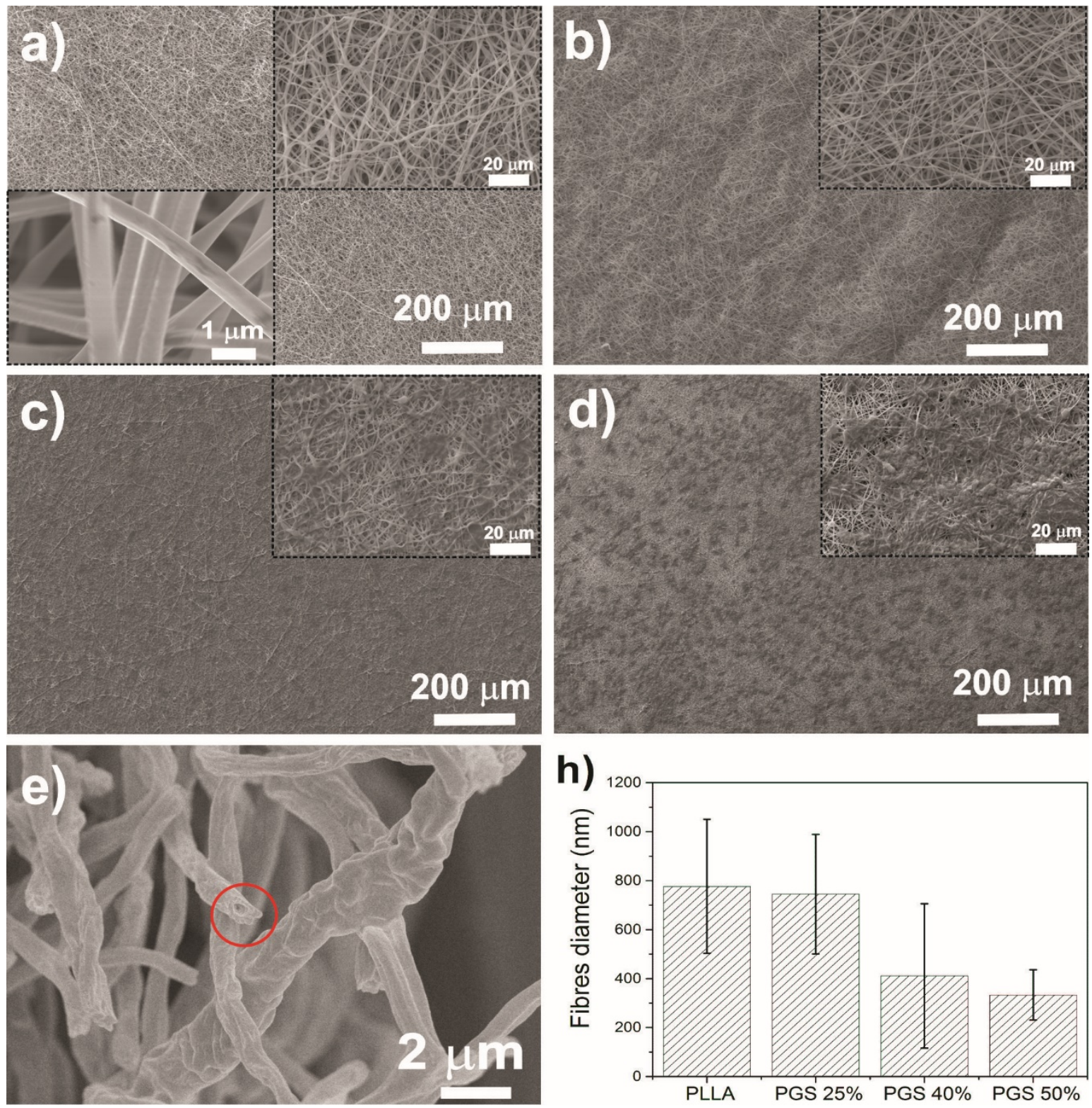

Figure 1: Electrospun sample surface morphology: a) PLLA annealed at $120{ }^{\circ} \mathrm{C}$ for $48 \mathrm{~h}$, b) cured sample with 25 wt\% PGS, c) cured sample with 40 wt\% PGS, d) cured sample with 50 wt\% PGS, (e) cross-section detail of the cured sample with $25 \mathrm{wt} \%$ PGS, and h) average fibre diameter \pm standard deviation, measured with image J. Insets of a), b), c) and d) show a higher magnification of the electrospun samples. 


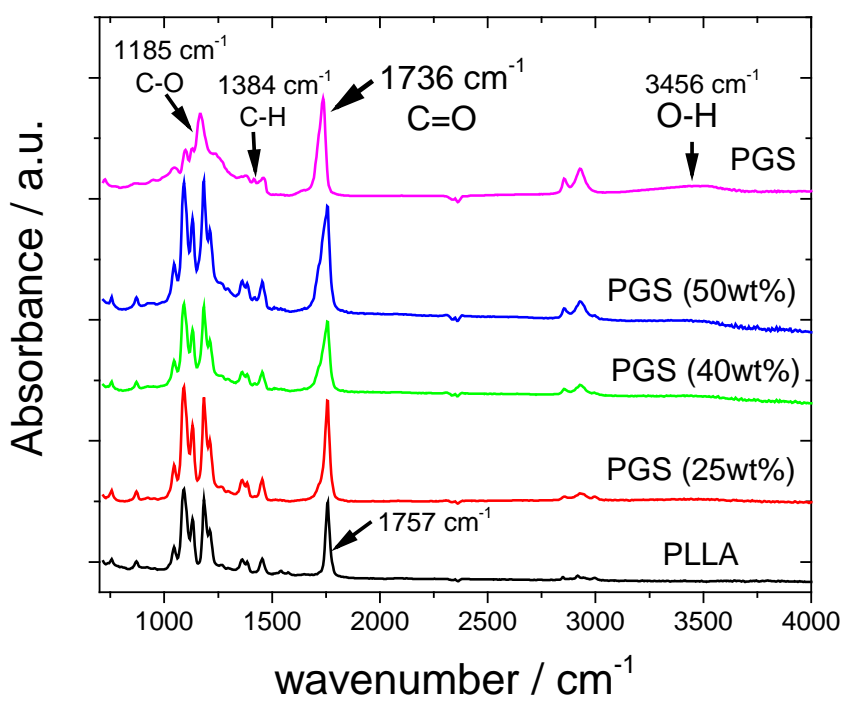

Figure 2: Infrared spectroscopy measurements recorded for the PLLA, PGS and PLLA:PGS samples. 

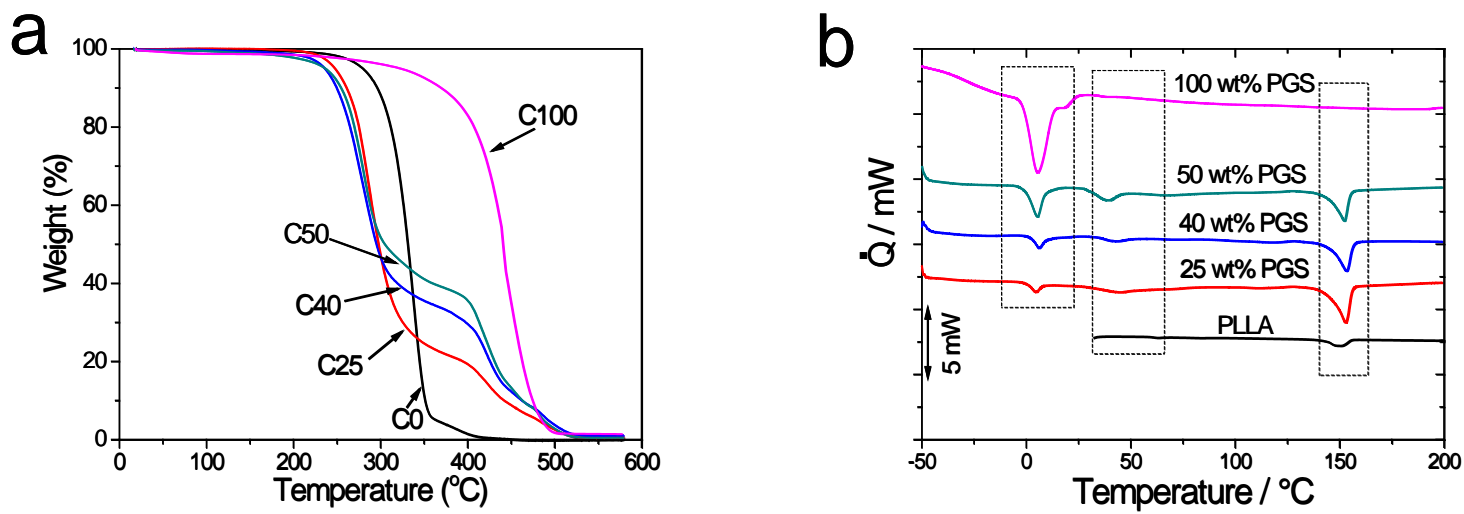

Figure 3: a) Thermogravimetric behavior, and b) differential scanning calorimetry recorded for the PLLA, PGS and PLLA:PGS core-shell fibres. 


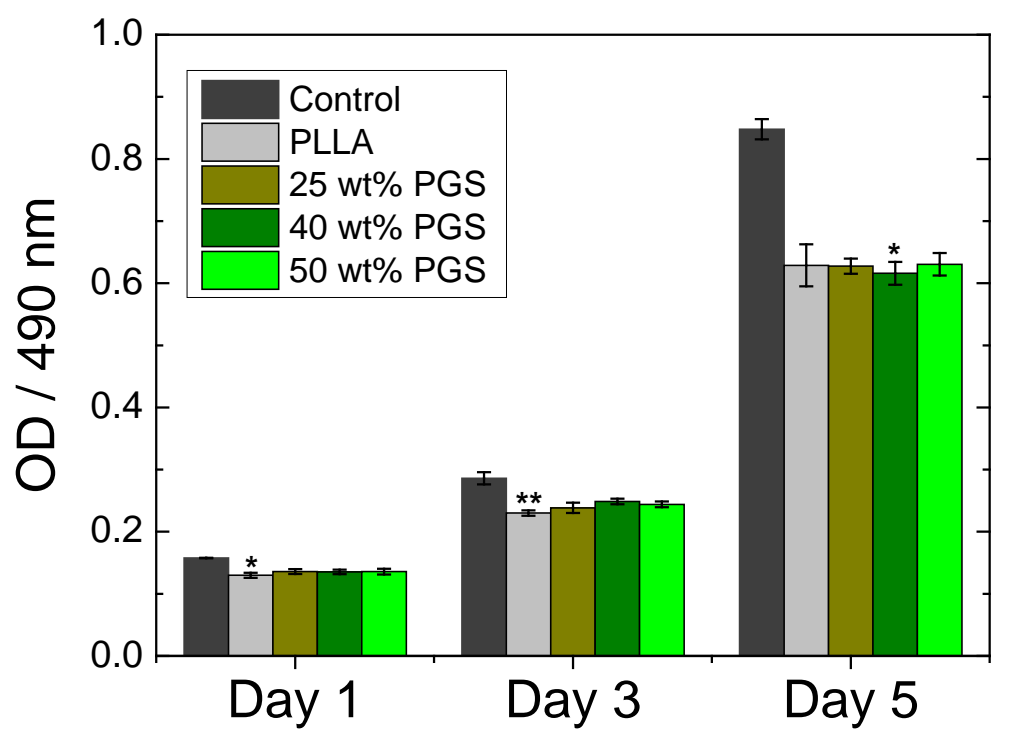

Figure 4: Proliferation of A59 neuronal cells cultured on PLLA, and PLLA:PGS electrospun membranes for 1,3 and 5 days. Statistical analysis: ${ }^{*} \mathrm{p}<0.05$. Results are presented as the mean \pm sd of three independent assays. Symbols $(*)$ denote statistical differences between the experimental groups and their respective controls (C) obtained by Kruskal-Wallis test with Dunn`s multiple comparison post-hoc test $(\mathrm{p}<0.05)$. 


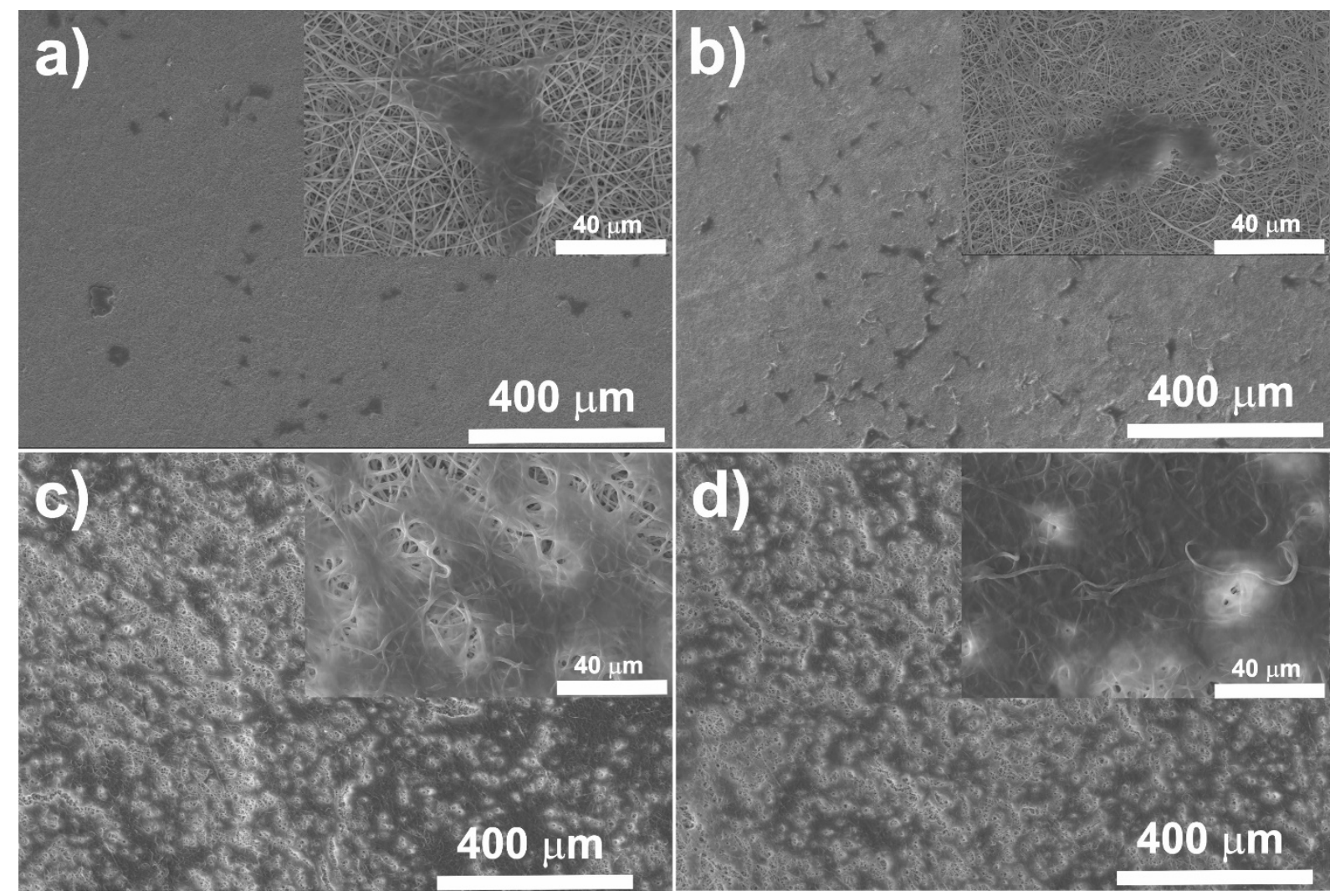

Figure 5: SEM micrographs of A59 neurons culture: 1 day cell culture on PLLA membrane (a); PLLA:PGS (25 wt\% PGS) membrane (b); 5 days PLLA cell culture (c) and 5 days cell culture on PLLA:PGS (25 wt\% PGS) membrane (d). 